Journal of Mathematics and Statistics 3 (4): 168-171, 2007

ISSN 1549-3644

(C) 2007 Science Publications

\title{
The Extended Laplace Transform
}

\author{
Sabra Ramadan \\ Department of Mathematics, Jazan University, Ministry of Higher Education \\ Jazan-Abu Areesh,P.O. Box 203, The Kingdom of Saudi Arabia
}

\begin{abstract}
The space of new generalized functions has been constructed. The operation of associative multiplication has been defined on this space. The Extended Laplace Transform has been defined.

Key words: New generalized functions, extended Laplace Transform, associative multiplication, semi norms, topology. 44A10,46F12
\end{abstract}

\section{INTRODUCTION}

If $f(t)$ is defined for $t \geq 0$, then the improper integral $\int_{0}^{\infty} K(s, t) f(t) d t$ has many important applications. The choice $K(s, t)=e^{-s t}$ gives us an especially important integral transform said to be Laplace transform of $f$, provided the integral converges.

In the linear mathematical model for a physical system such as a spring/mass system or a series electrical circuit, the right-hand member of the differential equation

$$
\begin{aligned}
& m \frac{d^{2} x}{d t^{2}}+\beta \frac{d x}{d t}+k x=f(t) \text { or } \\
& L \frac{d^{2} q}{d t^{2}}+R \frac{d q}{d t}+\frac{1}{C} q=E(t)
\end{aligned}
$$

is a driving function and represents either an external force $f(t)$ or an impressed voltage $E(t)$. Solving these differential equations in general is difficult but not impossible. The Laplace transform is an invaluable tool in solving problems such as these .

Moreover the Laplace transform can be used for solving the Voltera integral equations $f(t)+\lambda \int_{a}^{t} K(t, \tau) f(\tau) d \tau=g(t), \quad$ and $\quad$ integro differential equations $\quad D y=f(t)+\lambda \int_{a}^{t} y(\tau) K(t, \tau) d \tau$, where $D$ is the differential operator.
In (1) we defined the space $\zeta(\mathrm{E})$ as a factor space $T^{*}(E) / I^{*}(E){ }^{[5]}$ and we proved many Important results for this space. Also we have defined the extended Fourier Transform $\bar{F}: \zeta(\mathrm{S}(\mathrm{R})) \rightarrow \zeta(\mathrm{S}(\mathrm{R}))$.

Also in algebras $\xi(E)$ constructed in(1-5) all the operations of multiplication convolution, differentiation are defined.

There arises a natural question : How is to define the Laplace transform in those algebras?

In (6) the spaces $\Pi(R)$, and the space of New

Generalized functions $\zeta\left(\prod(R)\right)$ were constructed so that

$$
\Pi^{\prime}(R) \subset S^{\prime}(R) \subset \xi(S(R)) \subset \xi(\Pi(R)),
$$

where $\zeta(S(R))$ - the space of New Generalized functions constructed in (1); $S(R)$ - the space of test functions of rapid decay; $S^{\prime}(R)$ - the space of tempered distributions.

We also use the definitions, and some results in(6) .Lat us repeat some of them which are used throughout this paper .

Define the space $\Pi(R)=\prod_{1}(R) \cup \prod_{3}(R)$ where

$\prod_{1}=\left\{\eta(t) \in C^{\infty}(R): \lim _{t \rightarrow \infty} t^{n} \eta^{(k)}(t)=0, \forall n, k \in Z\right\}$

$\prod_{2}=\left\{\eta(t) \in C^{\infty}(0, \infty): \lim _{t \rightarrow \infty} t^{n} \eta^{(k)}(t)=0, \forall n, k \in Z\right\}$

$\Pi_{3}=\left\{g(t)=\eta(|t|): \eta(t) \in \Pi_{2}(R)\right\}$.

We define topology on $\Pi(R)$ by the following seminorms: 
$P_{\alpha}(\eta(t))=P_{n, l}(\eta(t))=\sup _{k \leq n, m \leq l} q_{k, m}(\eta(t))$ where $q_{k, m}(\eta(t))=\left.\sup _{\text {Theorem } \infty} 2 t^{k} \eta_{\text {Let }}^{(m)}(t)\right|_{\text {be an algebra satisfies (2.1) then }}$ The embedding of algebra $\zeta(S(R))$ in to the algebra $\zeta(\Pi(R))$ is defined by the following mapping:

$$
J_{\pi}:\left(\lambda_{k}\right)+I(S(R)) \rightarrow\left(\lambda_{k}\right)+I(\Pi(R))
$$

So we get the following results :

$$
\Pi^{\prime}(R) \subset S^{\prime}(R) \subset \zeta(S(R)) \subset \zeta(\Pi(R)) .
$$

In algebra $\zeta(\Pi(R))$ we define the associative multiplication for $\lambda=\left(\lambda_{k}\right)+I^{*}\left(\prod(R)\right)$,

$$
\begin{aligned}
& \gamma=\left(\gamma_{k}\right)+I^{*}(\Pi(R)) \text { by } \\
& \lambda \Theta \gamma=\left(\lambda_{k} \cdot \gamma_{k}\right)+I^{*}(\Pi(R)) .
\end{aligned}
$$

Theorem 1.1: The operation of multiplication $\Theta$ is independent of a representative .

Construction of $K(C)$ AND $\zeta(K(C))$

Let $K(a)$ be the set of all complex -valued functions analytic in the half plane $\operatorname{Re}(z) \geq a>0$, and

$\lim _{z \rightarrow \infty} F(z)=0$ in $-\frac{\pi}{2}+\delta<\arg (z)<\frac{\pi}{2}-\delta$ for each $\delta>0$.

Define the space $K(C)=\bigcup_{a \geq 0} K(a)$, and topology in this space define by the family of the following semi norms :

$$
\rho_{k}(F(z))=\sup _{\substack{\operatorname{Re}(z) \geq 0 \\ i \leq k}}\left|F^{(i)}(z)\right|
$$

By $T(E)$ we denote the set of all possible sequences in $\mathrm{E}$, where $\mathrm{E}$ be separated locally -convex algebra with topology defined by family of semi norms $\left(\mathrm{P}_{\alpha}\right)_{\alpha \in \mathrm{A}}$ such that for $\alpha \in A$, there exist $\beta \in A$ a constant $C_{\alpha}>0$ for which

$\rho_{\alpha}(\lambda . \gamma) \leq \mathrm{C}_{\alpha} \mathrm{P}_{\beta}(\lambda) \mathrm{P}_{\beta}(\gamma) \forall \lambda, \gamma \in \mathrm{E}$

Let $\mathrm{T}^{*}(\mathrm{E})$ be the set of all sequences $\left(\lambda_{\mathrm{k}}\right)_{\mathrm{k}=\mathrm{n}}^{\infty} \in \mathrm{E}$ satisfy the following conditions there is a number $\mathrm{m}$ such that for each $\alpha \in \mathrm{A}$, there is a nonnegative $\chi_{\alpha}>0$ such that $\mathrm{P}_{\alpha}\left(\lambda_{\mathrm{k}}\right) \leq \chi_{\alpha} \mathrm{k}^{\mathrm{m}}$ for each $\mathrm{k}$. And $I^{*}(E)$ be the set of all sequences $\left(\lambda_{k}\right)_{k=n}^{\infty} \in E$ satisfy the following conditions for each number $\mathrm{m}$ and for each $\alpha \in \mathrm{A}$, there is a nonnegative $\chi_{\alpha}>0$ such that $\mathrm{P}_{\alpha}\left(\lambda_{\mathrm{k}}\right) \leq \chi_{\alpha} \mathrm{k}^{-\mathrm{m}}$ for each $\mathrm{k}$. The following results are true: $\mathrm{T}^{*}(\mathrm{E})$ is a sub algebra of algebra $\mathrm{T}(\mathrm{E})$ and $\mathrm{I}^{*}(\mathrm{E})$ is an Ideal in $\mathrm{T}^{*}(\mathrm{E})$.

proof. see the prove in(1)

Theorem 2.2: The Space $K(C)$, with the topology given by the semi norms $\rho_{k}(F(z))=\sup _{\substack{\operatorname{Re}(z) \geq 0 \\ i \leq k}}\left|F^{(i)}(z)\right|$ satisfy the inequality (2.1).

proof: Consider $\rho_{k}(F(z) G(z))=\sup _{i \leq k}\left|(F G)^{(i)}(z)\right|$

$=\sup _{i \leq k}\left|\sum_{j=0}^{i} c_{i}^{j} F^{(j)}(z) \cdot G^{(i-j)}(z)\right| \leq$

$\leq \sup _{i \leq k}\left|(F)^{(i)}(z)\right| \sup _{i \leq k}\left|(G)^{(i)}(z)\right| \sup _{i \leq k}\left|\sum_{j=0}^{i} c_{i}^{j}\right| \leq$

$2^{i} \rho_{k}(F(z)) \rho_{k}(G(z))$.

We construct the algebra $\zeta(K(C))$ as a factor space $\zeta(K(C))=T^{*}(K(C)) / I^{*}(K(C))$.

If $L$ is the Laplace transform, then the image of the space $\Pi(R)$ is included in the space $K(C)$ (10), so we can write $L: \prod(R) \rightarrow K(C)$.

Theorem2.3: a. If $\lambda \in T^{*}\left(\prod(R)\right)$, then $L(\lambda(x)) \in T^{*}(K(C))$;

b. If $\lambda \in I^{*}\left(\prod(R)\right)$, then

$L(\lambda(x)) \in I^{*}(K(C))$.

Proof: Let $\lambda \in T^{*}(\Pi(R))$, then there is a number $\mathrm{m}$ such that for each $\alpha \in \mathrm{A}$, there is a nonnegative $\chi_{1}$ such that (2.2)

$p_{\alpha}\left(\lambda_{k}\right) \leq \chi_{1} k^{m}$ for each $\mathrm{k}$

From the continuity of the Laplace transform $L: \prod(R) \rightarrow K(C)$ implies that for each $j$ there is $i$ and a constant $c_{i}>0$, such that (2.3) $p_{j}\left(L\left(\lambda_{k}\right)\right) \leq c_{j 1} p_{i}\left(\lambda_{k}\right), \quad \forall k$.

Now from the inequalities (2.2), and (2.3) we see that there is $\mathrm{m}$ for each $j$ there $c_{j}=c_{j 1} \chi_{1}$ so that 
$p_{j}\left(L\left(\lambda_{k}\right)\right) \leq c_{j} k^{m}, \quad \forall k$. So we conclude that $L\left(\lambda_{k}\right) \in T^{*}\left(\prod(R)\right)$.

We can prove the second part in a similar way. Now from the proved theorem 2.3, and from the properties of the Laplace transform we conclude that the extended Laplace transform

$\tilde{L}: \zeta\left(\prod(R)\right) \rightarrow \zeta(K(R))$ is defined in the following way : Let $\lambda \in \zeta(\Pi(R))$, and let $\left(\lambda_{k}\right)$ be any representative for $\lambda$, then

$$
\tilde{L}(\lambda)=\left(L\left(\lambda_{k}\right)\right)+I^{*}(K(C)) .
$$

Theorem 2.4: The Laplace transform $\tilde{L}$ is independent of a representative .

Proof: Let $\lambda \in \zeta\left(\prod(R)\right)$, and let $\left(\lambda_{k}\right)$, and $\left(\gamma_{k}\right)$ be any two representatives for $\lambda$.That is $\left(\lambda_{k}-\gamma_{k}\right) \in I^{*}(\Pi(R))$ which means that for each number $\mathrm{m}$ and for each $\alpha \in \mathrm{A}$, there is a nonnegative $\chi_{1}$ such that (2.4)

$p_{\alpha}\left(\lambda_{k}-\gamma_{k}\right) \leq \chi_{1} k^{-m}$ for each $\mathrm{k}$

From the continuity of the Laplace transform $L: \prod(R) \rightarrow K(C)$ implies that for each $j$ there is $i$ and a constant $c_{i}>0$, such that (2.5) $p_{j}\left(L\left(\lambda_{k}-\gamma_{k}\right)\right) \leq c_{j 1} p_{\alpha}\left(\lambda_{k}-\gamma_{k}\right), \quad \forall k$

Now from the inequalities (2.4), and ( 2.5) we see that for each $j$ and for each $m$ there is $i$, and $c_{j}=c_{j 1} \chi_{1}$ so that $p_{j}\left(L\left(\lambda_{k}-\gamma_{k}\right)\right) \leq c_{j} k^{-m}, \forall k$.that is $\tilde{L}\left(\lambda_{k}-\gamma_{k}\right) \in I^{*}(K(C))$.

It is worthy to point here that the extended Laplace transform $\tilde{L}$ satisfy all properties of the ordinary Laplace transform (10):

Theorem 2.5: If $f, g \in \zeta\left(\prod(R)\right)$, and $\tilde{L} f=\tilde{F} \in \zeta(K(C)), \tilde{L} g=\tilde{G} \in \zeta(K(C))$, then

$$
\text { 1. } \tilde{L}\left[e^{p_{0} t} f(t)\right]=\tilde{F}\left(p-p_{0}\right)
$$

2. $\tilde{L}[(f * g)(t)]=\tilde{F}(p) \tilde{G}(p)$;

$$
\text { 3. } \tilde{L}[f(\alpha t)]=\frac{1}{\alpha} \tilde{F}\left(\frac{p}{\alpha}\right) ;
$$

4. $\tilde{L}\left[t^{n} f(t)\right]=(-1)^{n} \tilde{F}^{(n)}(p)$;

$$
\text { 5. } \tilde{L}\left[\int_{0}^{t} f(\tau) d \tau\right]=\frac{\tilde{F}(p)}{p}
$$

proof: 2. Let $f, g \in \zeta(\Pi(R))$, then

$$
\begin{aligned}
& f=\left(f_{k}\right)+I^{*}\left(\prod(R)\right), \text { and } \\
& g=\left(g_{k}\right)+I^{*}\left(\prod(R)\right) \text {.Consider } \\
& \tilde{L}[(f * g)(t)]=\left(L\left(f_{k}^{*} g_{k}\right)\right)+I^{*}\left(\prod(R)\right)= \\
& \left(L\left(f_{k}\right) \cdot L\left(g_{k}\right)\right)+I^{*}\left(\prod(R)\right)=\tilde{F}(p) \tilde{G}(p) .
\end{aligned}
$$

The prove of other properties is similar.

Moreover we get the following commutative diagram:

$$
\begin{array}{ccc}
\Pi(R) & \rightarrow \zeta(\Pi(R)) \\
L \downarrow & & \downarrow \tilde{L} \\
K(C) & \rightarrow & \zeta(K(C)) \\
& \beta &
\end{array}
$$

where $\alpha, \beta$ embeddings defined in the following way:

$$
\begin{array}{lll}
\alpha: \prod(R) \rightarrow \zeta(\Pi(R)), & \alpha(f)=\left(f_{k}\right) & \forall k ; \\
\beta: K(C) \rightarrow \zeta(K(C)), & \beta(g)=\left(g_{k}\right) & \forall k .
\end{array}
$$

So the following commutative formula is true :

$\tilde{L} o \alpha=\beta o L$.

\section{REFERENCES}

1. Radyno, Ya.V., Ngo Pho Tkan and Sabra Ramadan, 1993. Russian Acad. Sci. Dokl.327(1): American Mathematical Society. English Transl . in Russian Acad.Sci. Dokl. Math. Vol. 46 (1993),No.3:414-417

2. Radyno, Ya.V., A. V. Romashevskii and Sabra Ramadan, 1993 . Byelorussian Ac.Sci. Reports 
3. Ngo Pho Tkan and Sabra Ramadan, 1992. Weak Solutions of differential equations in Algebra of new generalized functions // Abstract of the conference of Belarus Mathematicians. Grodno 1992:112.

4. Sabra Ramadan and Ngo Pho Tkan, 1992. Convolution and Fourier Transform in Algebras of new generalized functions // Abstracts of the interrepuplican Scientific and practical conference of Young Scientific Workers. Minsk, 1992: 209.

5. Sabra Ramadan, 1998. The New Generalized Function Spaces $L(D(R))$, and $L(Z(R))$, Dirasat 25(1):20-24.
6. Sabra Ramadan,2007. One Algebra of New Generalized Functions.J.Math.\& Stat., Vol.3(1):1516,ISSN1549-3644.

7. Sabra Ramadan, 2007. Generalized Cauchy's Models and Generalized Integrals.J.Math.\& Stat., Vol.3(1):21-23,ISSN1549-3644.

8. Colombeau, J.E. 1984 , New generalized functions and multiplication of distribution, NorthHolland, Math. Studies. Amsterdam, N84.

9. Egorov,Ya.V., 1990. Uspekhi Math. Nauk 45 ( 5 ) : 3-40; English Transl. in Russian Math. Surveys. 45 (1990).

10. A.David Wunch, Complex Variables With Applications. 1994. 